\title{
Effect of thrombectomy on oedema progression and clinical outcome in patients with a poor collateral profile
} Gabriel Broocks (D) , ${ }^{1}$ Andre Kemmling, ${ }^{2,3}$ Tobias Faizy, ${ }^{4}$ Rosalie McDonough, ${ }^{1}$
Noel Van Horn, ${ }^{1}$ Matthias Bechstein, ${ }^{1}$ Lukas Meyer, ${ }^{1}$ Gerhard Schön, ${ }^{5}$
Jawed Nawabi, ${ }^{6}$ Jens Fiehler, ${ }^{1}$ Helge Kniep, ${ }^{1}$ Uta Hanning ${ }^{1}$

To cite: Broocks G, Kemmling A, Faizy T, et al. Effect of thrombectomy on oedema progression and clinical outcome in patients with a poor collateral profile. Stroke \& Vascular Neurology 2021;6: e000570. doi:10.1136/svn2020-000570

- Additional material is published online only. To view please visit the journal online (http://dx.doi.org/10.1136/svn2020-000570).

HK and UH contributed equally.

Received 8 August 2020

Revised 10 0ctober 2020

Accepted 23 October 2020

Published Online First

18 November 2020

Check for updates

(C) Author(s) (or their employer(s)) 2021. Re-use permitted under CC BY-NC. No commercial re-use. See rights and permissions. Published by BMJ.

For numbered affiliations see end of article.

Correspondence to Dr Gabriel Broocks; G.broocks@uke.de

\section{ABSTRACT}

Background and purpose The impact of the cerebral collateral circulation on lesion progression and clinical outcome in ischaemic stroke is well established. Moreover, collateral status modifies the effect of endovascular treatment and was therefore used to select patients for therapy in prior trials. The purpose of this study was to quantify the effect of vessel recanalisation on lesion pathophysiology and clinical outcome in patients with a poor collateral profile.

Materials and methods 129 patients who had an ischaemic stroke with large vessel occlusion in the anterior circulation and a collateral score (CS) of 0-2 were included. Collateral profile was defined using an established 5-point scoring system in CT angiography. Lesion progression was determined using quantitative lesion water uptake measurements on admission and follow-up CT (FCT), and clinical outcome was assessed using modified Rankin Scale (mRS) scores after 90 days. Results Oedema formation in FCT was significantly lower in patients with vessel recanalisation compared with patients with persistent vessel occlusion (mean 19.5\%, 95\% Cl: 17\% to $22 \%$ vs mean $27 \%, 95 \%$ Cl: $25 \%$ to $29 \%$; $p<0.0001$ ). In a multivariable linear regression analysis, vessel recanalisation was significantly associated with oedema formation in FCT ( $\beta=-7.31, S D=0.015, p<0.0001$ ), adjusted for $C S$, age and Alberta Stroke Program Early CT Score (ASPECTS). Functional outcome was significantly better in patients following successful recanalisation (mRS at day 90: 4.5, IQR: 2-6 vs 5, IQR: 5-6, p<0.001).

Conclusion Although poor collaterals are known to be associated with poor outcome, endovascular recanalisation was still associated with significant oedema reduction and comparably better outcome in this patient group. Patients with poor collaterals should not generally be excluded from thrombectomy.

\section{INTRODUCTION}

Mechanical thrombectomy in acute ischaemic stroke is of benefit to patients with a large vessel occlusion in the anterior circulation. ${ }^{12}$ The impact of cerebral collateral circulation on lesion progression and clinical outcome in ischaemic stroke is well established. ${ }^{3}$ Moreover, collateral status modifies the effect of endovascular treatment and was therefore used to select patients for therapy in prior studies. ${ }^{4}$ Although current guidelines do not recommend the exclusion of patients with poor collaterals from thrombectomy, the selection protocols for treatment still differed among the major thrombectomy trials. The ESCAPE and ESCPAE-NA1 trial, for example, specifically excluded this patient population from the endovascular treatment arm. ${ }^{45}$

Recently, it has been described that endovascular recanalisation is associated with oedema reduction, which was in contrast to prior studies observing higher oedema formation following revascularisation. ${ }^{6} 7$ However, the mechanisms of this phenomenon remain unclear. Considering the known relationship between the status of intracranial collaterals and ischaemic lesion water uptake, collateral status may be an important link between recanalisation and the effect on oedema formation. ${ }^{8}$ Yet, the impact of endovascular vessel recanalisation on oedema progression according to the collateral status remains unknown. A positive effect of endovascular treatment on oedema formation in patients with poor collaterals might have a direct impact on the occurrence of malignant infarctions with consecutive very poor outcome, which would deliver arguments for treatment despite poor intracranial collaterals.

The purpose of this study was (1) to quantify the effect of endovascular recanalisation in patients with a poor collateral profile on lesion pathophysiology measured by quantitative lesion water uptake; and (2) to compare clinical outcome in these patients according to the status of vessel recanalisation. We hypothesised that endovascular vessel recanalisation in patients with poor collaterals is associated with improved clinical outcome by directly altering lesion water homeostasis. 


\section{METHODS}

\section{Patients}

All patients who had an ischaemic stroke with acute large vessel occlusion in the anterior circulation admitted between February 2015 and March 2019 to our highvolume tertiary stroke centre were analysed. The study was conducted in accordance with the ethical guidelines of the local ethics committee and in accordance with the Declaration of Helsinki. The data that support the findings of this study are available from the corresponding author on reasonable request.

The a priori defined inclusion criteria were: (1) acute anterior circulation stroke in the territory of the middle cerebral artery (MCA) and MCA occlusion or distal occlusion of the internal carotid artery; (2) multimodal CT imaging protocol at admission including CT angiography (CTA) and CT perfusion (CTP); (3) a collateral score (CS) of 0-2 in admission CTA; (4) known time window from symptom onset to admission imaging within 6 hours; (5) follow-up CT (FCT) after 24 hours; (6) admission National Institutes of Health Stroke Scale (NIHSS) score $>3$; (7) absence of intracranial haemorrhage and preexisting thromboembolic or haemodynamic infarctions in admission non-enhanced CT (NECT) or pre-existing significant carotid stenosis; (8) absence of significant motion artefacts. Baseline patient characteristics were retrieved from the medical records, including modified Rankin Scale (mRS) after 90 days. Successful vessel recanalisation was defined as Thrombolysis in Cerebral Infarction Scale (TICI) 2b or 3.

\section{Image acquisitions}

All patients received multimodal stroke imaging at admission with NECT, CTA and CTP performed in equal order on 256 dual slice scanners (Philips iCT 256). NECT: 120 $\mathrm{kV}, 280-340 \mathrm{~mA}, 5.0 \mathrm{~mm}$ slice reconstruction, $1 \mathrm{~mm}$ increment; CTA: $100-120 \mathrm{kV}, 260-300 \mathrm{mAs}, 5.0 \mathrm{~mm}$ slice reconstruction, $1 \mathrm{~mm}$ increment, $80 \mathrm{~mL}$ highly iodinated contrast medium and $50 \mathrm{~mL} \mathrm{NaCl}$ flush at $4 \mathrm{~mL} / \mathrm{second}$; CTP: $80 \mathrm{kV}, 200-250 \mathrm{~mA}, 5 \mathrm{~mm}$ slice reconstruction (maximum $10 \mathrm{~mm}$ ), slice sampling rate $1.50 \mathrm{~s}$ (minimum $1.33 \mathrm{~s}$ ), scan time $45 \mathrm{~s}$ (maximum $60 \mathrm{~s}$ ), biphasic injection with $30 \mathrm{~mL}$ (maximum $40 \mathrm{~mL}$ ) of highly iodinated contrast medium with $350 \mathrm{mg}$ iodine $/ \mathrm{mL}$ (maximum $400 \mathrm{mg} / \mathrm{mL}$ ) injected with at least $4 \mathrm{~mL} / \mathrm{s}$ (maximum 6 $\mathrm{mL} / \mathrm{s}$ ) followed by $30 \mathrm{~mL}$ sodium chloride chaser bolus.

\section{Image analysis}

The anonymised imaging data was processed at an external core laboratory. Image analysis including volumetric and densitometric analysis was performed using commercially available software (Analyze 11.0, Biomedical Imaging Resource, Mayo Clinic, Rochester, Minnesota, USA).

Total lesion volume was measured in FCT using semiautomatic volumetric segmentation of the infarct lesion. Collateral status (CS) was assessed using an established 5-point scoring system. ${ }^{9}$ Intracranial CTA maximum intensity projections were used for scoring: $0=$ absent collaterals in $>50 \%$ of an MCA-M2 branch (superior or inferior division) territory; $1=$ diminished collaterals in $>50 \%$ of an MCA-M2 branch territory; 2=diminished collaterals in $<50 \%$ of an MCA-M2 branch territory; $3=$ collaterals equal to the contralateral hemisphere and $4=$ increased collaterals compared with the contralateral hemisphere. Good collaterals were defined as CS 3-4 and poor collaterals as CS $0-2$ according to Kim et al. ${ }^{10}$

Quantitative ischaemic lesion net water uptake (NWU) was determined as imaging biomarker to measure cerebral ischaemic oedema according to a published standardised procedure, as reported elsewhere. ${ }^{11-16}$ In admission NECT, the early hypoattenuated core lesion was assessed by densitometric measurements $\left(D_{\text {ischaemic }}\right)$. This region of interest (ROI) was mirrored to the contralateral hemisphere within normal tissue $\left(D_{\text {normal }}\right)$. CTP was used to improve the precision of ROI definition of the ischaemic core lesion by simultaneously presenting cerebral blood volume parameter maps at a fixed window between 0 and $6 \mathrm{~mL} / 100 \mathrm{~mL}$. ROIs in NECT were segmented with semiautomatic edge detection and sampled between intensity values of 20-80 Hounsfield Units (Analyze 12.0, AnalyzeDirect). Both density measurements $\left(D_{\text {normal }}\right.$ and $D_{\text {ischaemic }}$ ) were then used to calculate quantitative NWU $\left(\%\right.$ NWU $\left.=1-\left(D_{\text {ischaemic }} / D_{\text {normal }}\right) \times 100\right)$.

In FCT, NWU was quantified by defining $D_{\text {ischaemic }}$ using semiautomatic segmentation of the infarct lesion, as published. ${ }^{12}$ Figure 1 illustrates the impact of vessel recanalisation on lesion progression in patients with poor collaterals.

\section{Statistical analysis}

Kolmogorov-Smirnov tests were used to determine whether data were well-modeled by a normal distribution. Variables were presented as means with SD or medians with IQRs. Student's t-tests (normal distribution) with SD or Mann-Whitney U tests (non-normal distribution) with IQR were used to assess differences of the acquired parameters separately for patients with vessel recanalisation versus persistent vessel occlusion. The impact of age, NIHSS, ASPECTS, recanalisation status, NWU at admission and CS on functional outcome was investigated using univariable and multivariable logistic regression analysis (online supplemental table I) with backward selection and functional independence (mRS 0-2 after 90 days) as dependent variable.

Additionally, the impact of recanalisation and collateral status on clinical outcome was investigated and displayed using multivariable linear regression analysis with mRS at 90 days at dependent variable, as logistic regression analysis may be underpowered due to the expectedly low number of patients with poor collaterals, persistent vessel occlusion and good clinical outcome (figure 2). Among others, Sajobi et $a l^{17}$ have exemplified that ordinal 90-day mRS outcome measures may be treated as scale variable and employed in linear regression models. Univariable and multivariable linear regression analysis 


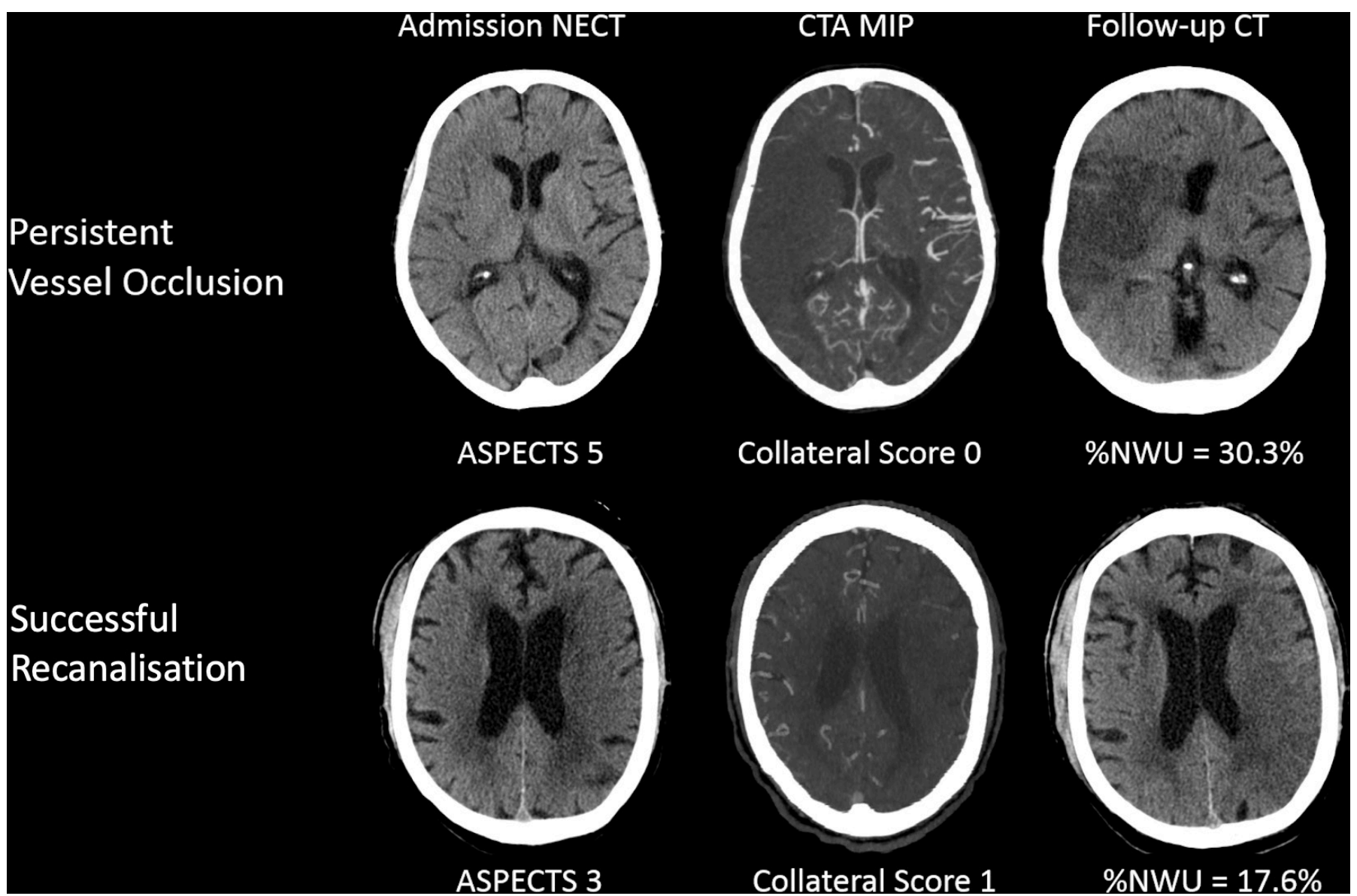

Figure 1 Illustration of recanalisation versus persistent vessel occlusion in two patients. Both patients presented with a low Alberta Stroke Program Early CT Score (ASPECTS) and poor collateral status at admission. The patient in the upper row did not undergo endovascular treatment and exhibited a significantly aggravated oedema formation in follow-up imaging. The patient in the lower row presented with an even lower ASPECTS of 3, but showed less oedema formation in follow-up imaging. CTA,CT angiography; MIP, maximum intensity projection; NECT, non-enhanced CT; NMU, net water uptake.

was performed to test the impact of CS, ASPECTS, age, recanalisation status and intravenous alteplase on total lesion volume and ischaemic lesion water uptake in FCT with backward selection (online supplemental table I), figure 2).

A statistically significant difference was accepted at a $p$ value of $<0.05$. Analyses were performed using MedCalc (V.11.5.1.0; Mariakerke, Belgium), Python seaborn 0.9.0 (Michael Waskom) and R (R Core Team, R: A Language and Environment for Statistical Computing, R Foundation for Statistical Computing, Vienna, Austria, 2017).

\section{Data availability statement}

The data that support the findings of this study are available from the corresponding author on reasonable request.

\section{RESULTS}

One hundred and twenty-nine patients with a CS of 0-2 were included (CS $0=37$; CS $1=50 ; \operatorname{CS~2=42).~The~mean~}$ (SD) age of these patients was 73 (12), and the median NIHSS was 18 (IQR: 15-20). Admission imaging was performed after a median time of 3 hours after symptom onset (IQR: 2-4). The median initial ASPECTS was 5 (IQR: 4-8). Patients' characteristics are shown in table 1.

One hundred and four $(81 \%)$ patients with poor collaterals received mechanical thrombectomy, of which 87 patients received successful vessel recanalisation (TICI $\geq 2$ b). Forty-two patients had persistent vessel occlusion: for 17 patients, a TICI $<2$ b was observed after thrombectomy, 14 patients did not receive intravenous lysis or an endovascular procedure and were assigned to the persistent vessel occlusion group and 11 patients received intravenous lysis but not endovascular procedure. In these patients, a persisting vessel occlusion was confirmed via dense artery sign in FCT and/or transcranial colour-coded duplex ultrasonography. ${ }^{18}$ Table 1 shows patient characteristics for the study cohort separated by status of vessel recanalisation.

\section{Clinical outcome}

Median mRS for patients with vessel recanalisation was 4.5 (IQR: 2-6), which was significantly different from the median mRS of patients with persistent vessel occlusion (median 5, IQR: 5-6; $\mathrm{p}<0.001$ ). Within the study cohort, there was one patient with poor collaterals and persistent 


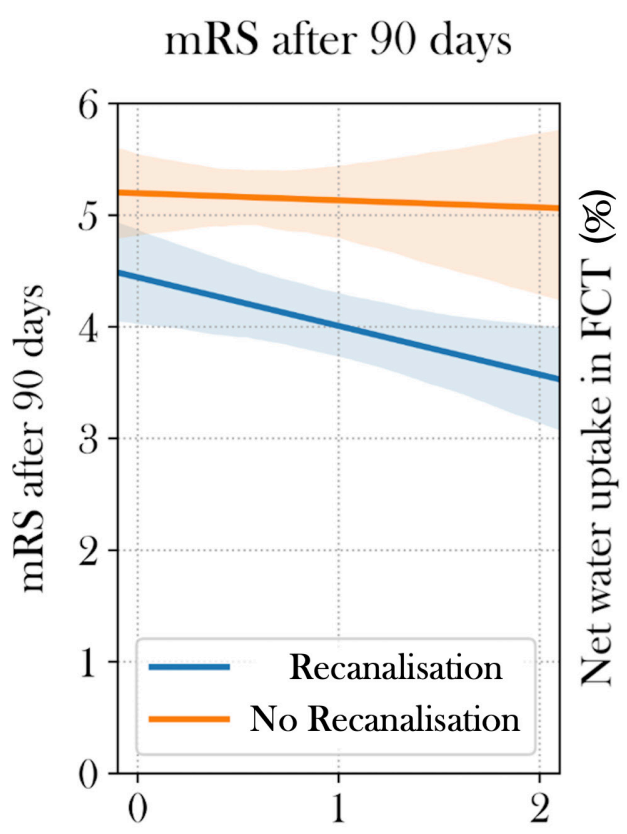

Collateral score
Water uptake FCT

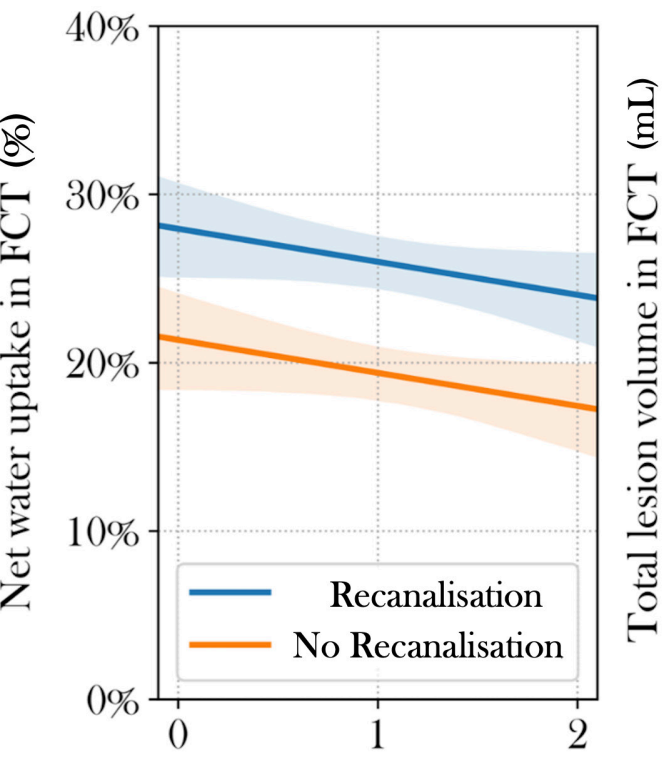

Collateral score
Lesion volume FCT

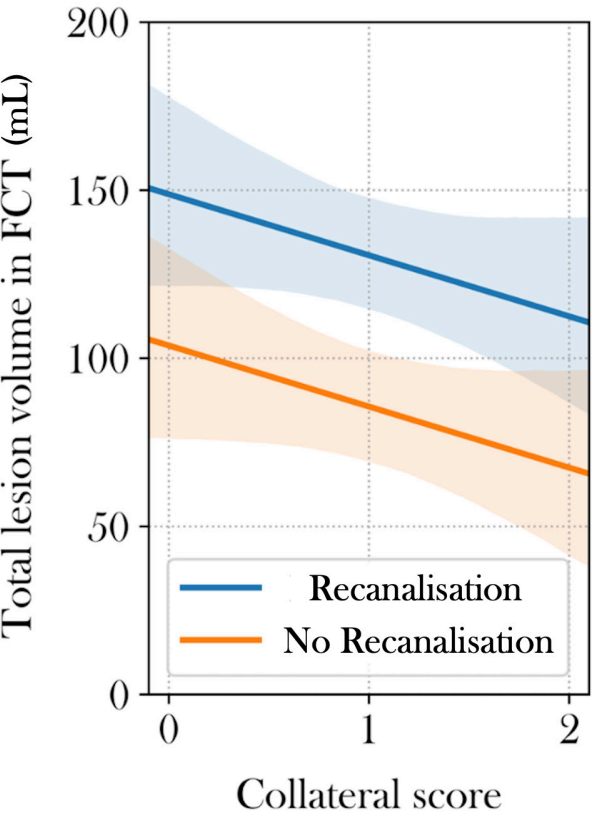

Figure 2 Regression analyses for clinical outcome and lesion pathophysiology. The impact of endovascular recanalisation in patients with poor intracranial collaterals (X axis) on modified ranking scale (mRS) score at 90 days (left), ischaemic lesion water uptake (middle) and total lesion volume (right) based on multivariable linear regression analysis. Ordinal mRS values were treated as scaled variable for linear regression. FCT, follow-up CT.

vessel occlusion with functional independence after 90 days (mRS 2), while there were 16 patients $(8 / 52)$ receiving vessel recanalisation who reached functional independence after 90 days (online supplemental figure I).

Univariable and multivariable logistic regression models were used to investigate the impact of baseline parameters and treatment on clinical outcome for patients with poor collaterals (CS 0-2). In univariable logistic regression analysis, CS, early lesion water uptake, ASPECTS, NIHSS, age, vessel recanalisation and application of intravenous lysis were associated with functional independence after 90 days (online supplemental table I). In multivariable logistic regression analysis, age, NIHSS, ASPECTS, CS and early lesion water uptake were significantly and independently associated with functional independence, adjusted for recanalisation status and application of intravenous lysis (online supplemental table I). In a model without ASPECTS, recanalisation was significantly associated with functional independence (OR 14.6, 95\% CI: 1.6 to 133.3 ; $\mathrm{p}=0.02$ ), adjusted for early lesion water uptake, NIHSS and age (figure 3). In multivariable linear regression analysis, successful recanalisation was associated with a significantly lower $\mathrm{mRS}$ at 90 days for patients with CS of 1 and 2 (online supplemental table I, figure 2).

\section{Total lesion volume in follow-up imaging}

Total lesion volume in FCT was $149.7 \mathrm{~mL}$ (IQR: 89.7$206.6 \mathrm{~mL}$ ) for patients with persistent vessel occlusion, which was significantly higher than lesion volume in FCT in patients with vessel recanalisation (median $43.3 \mathrm{~mL}$, IQR: 15.4-152.8 mL; $\mathrm{p}=0.0001$ ) (figure 3).
In multivariable linear regression analysis for patients with poor collaterals, vessel recanalisation $(B:-48 \mathrm{~mL}$, SD 19.4, $\mathrm{p}=0.01$ ), ASPECTS ( $(:-14 \mathrm{~mL}, \mathrm{SD} 4.1, \mathrm{p}=0.001$ ) and age $(B:-1.6 \mathrm{~mL}, \mathrm{SD} 0.7, \mathrm{p}=0.02)$ were significantly associated with total lesion volume in FCT, adjusted for collateral status (figure 2)

\section{Lesion water uptake in follow-up imaging}

Ischaemic lesion water uptake for patients with poor collaterals measured in FCT after treatment was $26.8 \%$ in patients with persistent vessel occlusion (95\% CI: $24.6 \%$ to $28.9 \%)$ and $19.5 \%$ in patients with vessel recanalisation (95\% CI: $17.3 \%$ to $21.6 \%)$, which was significantly different $(\mathrm{p}<0.0001)$ (figure 3). In univariable linear regression analysis for patients with poor collaterals (CS 0-2), vessel recanalisation, CS and ASPECTS were significantly associated with lesion water uptake in FCT (online supplemental table I). In multivariable linear regression analysis, vessel recanalisation was significant ( $B:-7.31 \%$, SD $0.015, \mathrm{p}<0.0001)$ and CS by trend $(B:-1.8 \%$, SD $1.0, \mathrm{p}=0.08)$ associated with lesion water uptake in FCT (online supplemental table I, figure 2).

\section{DISCUSSION}

The purpose of this study was to quantify the effect of endovascular recanalisation in patients with a poor collateral profile on lesion pathophysiology measured by quantitative lesion water uptake and to compare clinical outcome in these patients according to the status of vessel recanalisation. The main finding of this 


\section{Recanalisation in patients with poor collaterals}
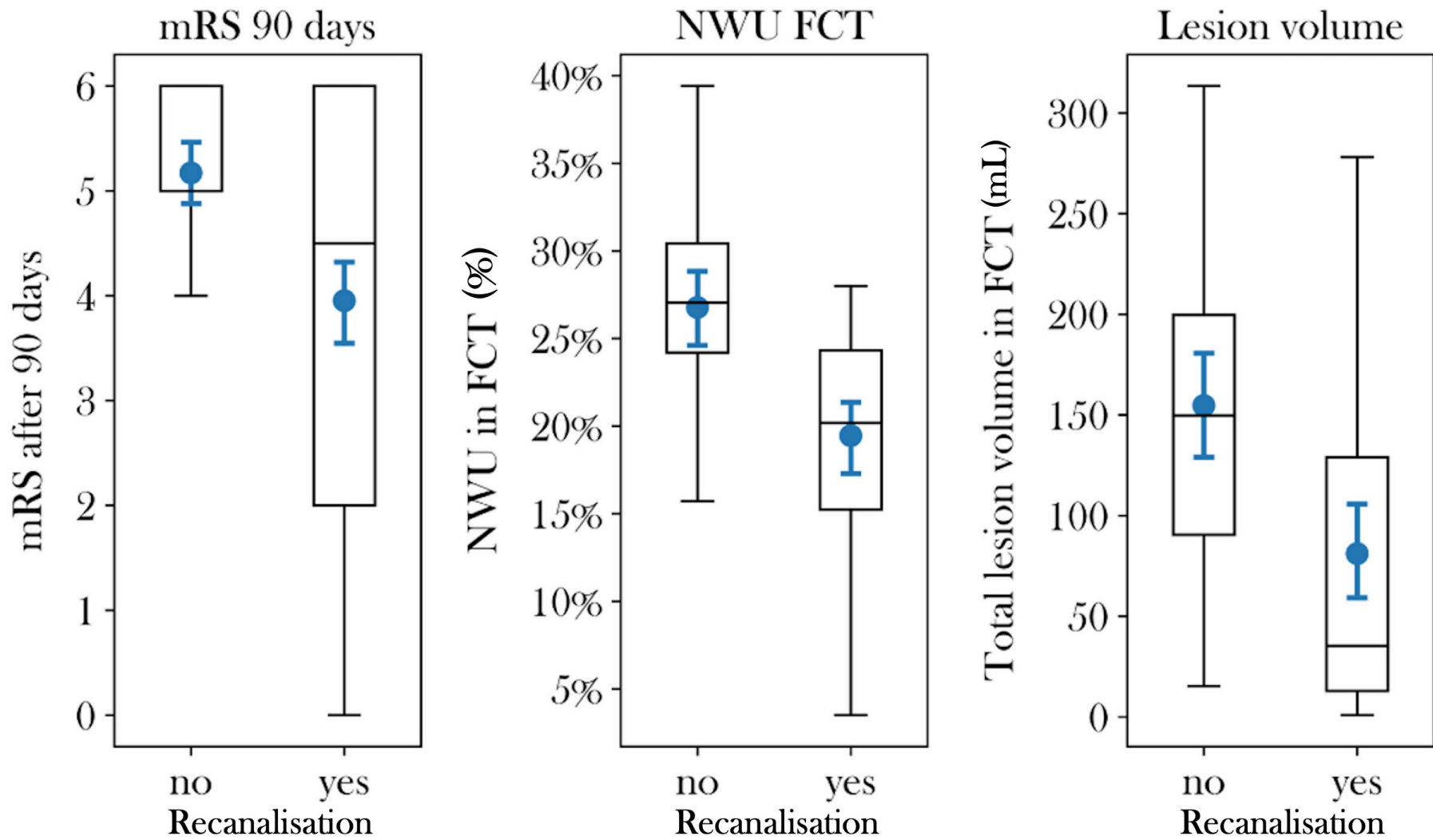

Figure 3 Clinical outcome, quantitative lesion water uptake and total lesion volume in the study cohort. Boxplots to illustrate differences in clinical outcome, indicated by modified ranking scale scores (mRS) at 90 days (left), per cent lesion water uptake in follow-up CT (FCT, middle) and total lesion volume (right) in patients with successful vessel recanalisation versus persistent vessel occlusion. NWU, netwater uptake.

study is that despite poor intracranial collaterals, endovascular recanalisation was associated with improved clinical outcome. The effect of vessel recanalisation on ischaemic lesion water homeostasis using a quantitative imaging biomarker was $-7.3 \%$ in this patient cohort with poor collaterals, which was similar to that of a previously described cohort consisting of patients with a range of collaterals scores $(-6.3 \%){ }^{12}$ The results of this study emphasise that patients with poor intracranial collaterals, who are often excluded in stroke trials, may still benefit from thrombectomy, with improved clinical outcome. ${ }^{19}$ Although the effect of vessel recanalisation on oedema formation has already been discussed, the exact mechanisms of this phenomenon remain uncertain. Factors such as age or blood glucose concentration have been discussed as important mediators of oedema formation, but the potential role of collaterals has not yet been described. ${ }^{1020} 21$ The status of the collateral circulation may be of particular importance in patients with a low initial ASPECTS, where a treatment benefit remains uncertain. Selection for endovascular treatment in this patient subgroup is therefore a controversial topic of discussion. ${ }^{1}$ A previous study described the proportion of low ASPECTS patients with functional independence to be approximately $20 \% .{ }^{13}$ Considering that $20 \%-30 \%$ of all low ASPECTS patients exhibit good collaterals, one might assume that only low ASPECTS patients with good collaterals benefit from endovascular treatment, especially regarding oedema reduction. ${ }^{22}{ }^{23}$ Conversely, the results of the present study suggest that vessel recanalisation in patients with poor collaterals results in a similar, or perhaps even higher, effect on oedema formation compared with those with good collaterals. Regarding the underlying pathophysiology, it is known that good collaterals allow for maintained penumbral perfusion, which has been linked to lower rates of oedema formation and hence lower interstitial pressure and lower resistance of collateral and downstream perforating arterioles. In patients with poor collaterals, on the contrary, cerebral blood flow logically decreases faster and to a greater extent, resulting in elevated interstitial pressure and thereby contributing to more rapid aggravation of ischaemic oedema and expansion of the ischaemic core. ${ }^{24}$ The lower persisting blood flow in patients with poor collaterals that results in higher oedema formation over time may explain the comparably higher impact of vessel recanalisation on oedema formation in this patient group. As the tissue-at-risk is not supplied by significant collateralisation, its (re)perfusion mainly depends on the occluded vessel itself. Hence, the relative effect of 
Table 1 Characteristics of patients with poor collaterals $(n=129)$

\begin{tabular}{llcl}
\hline Patient characteristics & $\begin{array}{l}\text { Vessel } \\
\text { recanalisation }\end{array}$ & $\begin{array}{l}\text { Persistent vessel } \\
\text { occlusion }\end{array}$ & $\begin{array}{l}\text { Group comparison } \\
\text { P value }\end{array}$ \\
\hline Subjects, $\mathrm{n}(\%)$ & $87(67)$ & $42(33)$ & na \\
\hline Age in years, median (IQR) & $76(65-82)$ & $73(69-84)$ & 0.62 \\
\hline Female sex, $\mathrm{n}(\%)$ & $36(42)$ & $17(41)$ & 0.89 \\
\hline Admission NIHSS, median (IQR) & $17(15-20)$ & $19(17-20)$ & 0.03 \\
\hline ASPECTS, median (IQR) & $6(5-8)$ & $4(3-5)$ & $<0.001$ \\
\hline Time onset to imaging in hours, mean (SD) & $2.9(2.1)$ & $3.7(1.6)$ & 0.03 \\
\hline Mechanical thrombectomy, $\mathrm{n}(\%)$ & $87(100)$ & $17(41)$ & $<0.01$ \\
\hline Collateral Score, median (IQR) & $1(1-2)$ & $1(0-1)$ & 0.003 \\
\hline Initial NWU in \%, mean (SD) & $11.0(5.6)$ & $10.6(4.3)$ & 0.69 \\
\hline Follow-up endpoints & & & \\
\hline Follow-up infarct volume in mL, median (IQR) & $35(13-129)$ & $150(90-206)$ & $<0.001$ \\
\hline Follow-up NWU in \%, mean (SD) & $19.5(6.0)$ & $26.8(6.4)$ & $<0.001$ \\
\hline mRS, median (IQR) & $4.5(2-6)$ & $5(5-6)$ & $<0.001$ \\
\hline mRS 0-2, $\mathrm{n}(\%)$ & $23(26)$ & $1(2)$ & $<0.001$ \\
\hline mRS 3-4, $\mathrm{n}(\%)$ & $19(22)$ & $7(17)$ & 1.0 \\
\hline mRS 5-6, $\mathrm{n}(\%)$ & $42(48)$ & $33(79)$ & $<0.001$ \\
\hline${ }^{*}$ mRS score not available, $\mathrm{n}$ & 3 & 1 & \\
\hline
\end{tabular}

mRS, modified Rankin Scale; NIHSS, National Institutes of Health Stroke Scale; NWU, net water uptake.

vessel recanalisation on perfusion leading to oedema reduction could be higher in patients with poor collaterals.

The positive effect of thrombectomy on clinical outcome in this patient group may be caused by oedema reduction, as previously described, although thrombectomy was not significantly associated with functional independence in a multivariable model that included ASPECTS. ${ }^{13}$ On admission imaging, only 23/129 (17.8\%) with poor collaterals showed an ASPECTS of 7-10, which may emphasise why endovascular recanalisation was not significantly associated with functional independence in a multivariable model that included ASPECTS. Therefore, the constellation of poor collaterals and higher ASPECTS, which occurred in nearly $20 \%$ of patients with poor collaterals in this cohort should be interpreted as a call of rapid endovascular treatment, especially in patients within a hyperacute time window, according to the results of this study. ${ }^{25}$

To our knowledge, this is the first study that investigated the impact of vessel recanalisation on lesion progression and clinical outcome in patients with poor collaterals, and how recanalisation may be of benefit in this patient group. Second, we observed that recanalisation was not only associated with reduced total lesion volume but also reduced ischaemic lesion water uptake in FCT, which was determined using a quantitative imaging biomarker. The effect of revascularisation on oedema formation might result in reduced occurrence of malignant infarctions, which could be the link to improved outcome in this patient group.
Collateral status remained to be significantly and independently associated with clinical outcome and by trend with oedema formation in multivariable analysis (statistically significant in univariable analysis), despite preselecting only patients with a lower CS of $0-2$. This may suggest significant differences within the lower CSs. Regarding selection for treatment, patients with poor to intermediate collaterals should not be generally excluded from treatment, as mentioned above. Instead, a very poor CS, previously described as 'malignant' could be used as a more specific tool to exclude patients from treatment, especially in a scenario of admission to a primary stroke centre without an opportunity of rapid endovascular treatment ${ }^{26}$ (see figure 2). Figure 2 exemplifies how vessel recanalisation was significantly associated with improved mRS score at 90 days in patients with CS 1 and 2, while CIs overlapped in patients with CS 0 .

Limitations of our study include the retrospective nature of this study and the relatively small number of patients, mainly caused by strict inclusion criteria to obtain a homogeneous patient cohort. Fourteen patients did not receive intravenous lysis or an endovascular procedure and were assigned to the persistent vessel occlusion group. In this group of patients, spontaneous recanalisation might have occurred, and therefore wrongly assigned. However, the rate of spontaneous recanalisation in large vessel occlusion stroke is low and has been described as being around $4 \%-20 \% .{ }^{27} 28$ Therefore, the number of patients incorrectly matched is considered to be very low in this patient cohort. Finally, different classifications and techniques of 
scoring the status of intracranial collaterals could affect comparability.

\section{CONCLUSION}

In patients with poor collaterals, endovascular recanalisation was associated with a significant oedema reduction and improved clinical outcome. Therefore, this group of patients should not generally be excluded from endovascular treatment. Alternatively, a 'malignant' CS of 0 could be used as selection criterion.

\section{Author affiliations}

${ }^{1}$ Department of Diagnostic and Interventional Neuroradiology, University Medical Center Hamburg-Eppendorf, Hamburg, Germany

${ }^{2}$ Institute of Neuroradiology, University Hospital of Luebeck, Luebeck, Germany ${ }^{3}$ Neuroradiology, Westpfalzklinikum, Kaiserslautern, Germany

${ }^{4}$ Department of Diagnostic and Interventional Neuroradiology, Stanford University, Stanford, California, USA

${ }^{5}$ Department of Medical Biometry and Epidemiology, University Medical Center Hamburg Eppendorf, Hamburg, Germany

${ }^{6}$ Department of Radiology, Charité Universitätsmedizin Berlin, Berlin, Germany

Contributors GB contributed to study design, acquisition of data, image processing, data analysis, statistical analysis, drafting the manuscript and revising it critically. AK contributed to data analysis, acquisition of data, drafting the manuscript and revising it critically. TF contributed to data analysis, statistical analysis and acquisition of data. RM / NvH: contributed to image processing, statistical analysis and acquisition of data. MB contributed to data analysis, acquisition of data, drafting the manuscript and revising it critically. LM contributed to data analysis, drafting the manuscript and revising it critically. GS contributed to data analysis and statistical analysis. JN contributed to acquisition of data, data analysis and image processing. JF contributed to study design, drafting the manuscript and revising it critically. HK contributed to study design, drafting the manuscript and revising it critically. UH contributed to study design, acquisition of data, image processing, data analysis, statistical analysis, drafting the manuscript and revising it critically.

Funding The authors have not declared a specific grant for this research from any funding agency in the public, commercial or not-for-profit sectors.

Competing interests JF: Research support: German Ministry of Science and Education (BMBF), German Ministry of Economy and Innovation (BMWi), German Research Foundation (DFG), European Union (EU), Hamburgische Investitionsund Förderbank (IFB), Medtronic, Microvention, Route92, Stryker. Consultant for: Acandis, Bayer, Boehringer Ingelheim, Cerenovus, Evasc Neurovascular, MD Clinicals, Medtronic, Microvention, Penumbra, Phenox, Stryker, Transverse Medical. Stock holder: Tegus Medical.

Patient consent for publication Not required.

Ethics approval This study has been approved by the local ethics committee (Ethikkommission der Ärztekammer Hamburg). Informed consent has been waived. Provenance and peer review Not commissioned; externally peer reviewed. Data availability statement Data that support the findings of this study are available upon reasonable request.

Open access This is an open access article distributed in accordance with the Creative Commons Attribution Non Commercial (CC BY-NC 4.0) license, which permits others to distribute, remix, adapt, build upon this work non-commercially, and license their derivative works on different terms, provided the original work is properly cited, appropriate credit is given, any changes made indicated, and the use is non-commercial. See: http://creativecommons.org/licenses/by-nc/4.0/.

ORCID iD

Gabriel Broocks http://orcid.org/0000-0002-7575-9850

\section{REFERENCES}

1 Román LS, Menon BK, Blasco J, et al. Imaging features and safety and efficacy of endovascular stroke treatment: a meta-analysis of individual patient-level data. Lancet Neurol 2018;17:895-904.
2 Goyal M, Menon BK, van Zwam WH, et al. Endovascular thrombectomy after large-vessel ischaemic stroke: a meta-analysis of individual patient data from five randomised trials. Lancet 2016;387:1723-31.

3 Boers AM, Jansen IG, Berkhemer OA, et al. Collateral status and tissue outcome after intra-arterial therapy for patients with acute ischemic stroke. J Cereb Blood Flow Metab 2017;37:3589-98.

4 Goyal M, Demchuk AM, Menon BK, et al. Randomized assessment of rapid endovascular treatment of ischemic stroke. $N$ Engl J Med 2015;372:1019-30.

5 Hill MD, Goyal M, Menon BK, et al. Efficacy and safety of nerinetide for the treatment of acute ischaemic stroke (ESCAPE-NA1): a multicentre, double-blind, randomised controlled trial. Lancet 2020;395:878-87.

6 Pillai DR, Dittmar MS, Baldaranov D, et al. Cerebral ischemiareperfusion injury in rats--a 3 T MRI study on biphasic blood-brain barrier opening and the dynamics of edema formation. $J$ Cereb Blood Flow Metab 2009;29:1846-55.

7 Broocks G, Hanning U, Flottmann F, et al. Clinical benefit of thrombectomy in stroke patients with low aspects is mediated by oedema reduction. Brain 2019;142:1399-407.

8 Broocks G, Kemmling A, Meyer L, et al. Computed tomography angiography collateral profile is directly linked to early edema progression rate in acute ischemic stroke. Stroke 2019;50:3424-30.

9 Souza LCS, Yoo AJ, Chaudhry ZA, et al. Malignant cta collateral profile is highly specific for large admission DWI infarct core and poor outcome in acute stroke. AJNR Am J Neuroradiol 2012;33:1331-6.

$10 \mathrm{Kim}$ J-T, Liebeskind DS, Jahan R, et al. Impact of hyperglycemia according to the collateral status on outcomes in mechanical thrombectomy. Stroke 2018;49:2706-14.

11 Broocks G, Flottmann F, Ernst M, et al. Computed tomographybased imaging of Voxel-Wise lesion water uptake in ischemic brain: relationship between density and direct volumetry. Invest Radiol 2018;53:207-13.

12 Broocks G, Flottmann F, Hanning U, et al. Impact of endovascular recanalization on quantitative lesion water uptake in ischemic anterior circulation strokes. J Cereb Blood Flow Metab 2020;40:437-45.

13 Broocks G, Hanning U, Flottmann F, et al. Clinical benefit of thrombectomy in stroke patients with low aspects is mediated by oedema reduction. Brain 2019;142:1399-407.

14 Minnerup J, Broocks G, Kalkoffen J, et al. Computed tomographybased quantification of lesion water uptake identifies patients within 4.5 hours of stroke onset: a multicenter observational study. Ann Neurol 2016;80:924-34.

15 Nawabi J, Flottmann F, Hanning U, et al. Futile recanalization with poor clinical outcome is associated with increased edema volume after ischemic stroke. Invest Radiol 2019;54:282-7.

16 Broocks $\mathrm{G}$, Leischner $\mathrm{H}$, Hanning $\mathrm{U}$, et al. Lesion Age Imaging in Acute Stroke: Water Uptake in CTVersus DWI-FLAIRMismatch. Ann Neurol 2020;49. [Epub ahead of print: 16 Sep 2020].

17 Sajobi TT, Zhang Y, Menon BK, et al. Effect size estimates for the escape trial: proportional odds regression versus other statistical methods. Stroke 2015;46:1800-5.

18 Minnerup J, Wersching $\mathrm{H}$, Ringelstein EB, et al. Prediction of malignant middle cerebral artery infarction using computed tomography-based intracranial volume reserve measurements. Stroke 2011;42:3403-9.

19 Broocks G, Kniep H, Schramm P, et al. Patients with low Alberta stroke program early CT score (aspects) but good collaterals benefit from endovascular recanalization. J Neurointerv Surg 2020;12:747-52.

20 Broocks G, Kemmling A, Aberle J, et al. Elevated blood glucose is associated with aggravated brain edema in acute stroke. $J$ Neurol 2020;267:440-8.

21 Meyer L, Schönfeld M, Bechstein M, et al. Ischemic lesion water homeostasis after thrombectomy for large vessel occlusion stroke within the anterior circulation: the impact of age. J Cereb Blood Flow Metab 2020;271678X:20915792.

22 Tan BYQ, Wan-Yee K, Paliwal P, et al. Good intracranial collaterals Trump poor aspects (Alberta stroke program early CT score) for intravenous thrombolysis in anterior circulation acute ischemic stroke. Stroke 2016;47:2292-8.

23 Broocks G, Fiehler J, Kemmling A. Collateral scoring in acute stroke patients with low aspects: an unnecessary or underestimated tool for treatment selection? Brain 2019;142:e36.

24 Rocha M, Jovin TG. Fast versus slow Progressors of infarct growth in large vessel occlusion stroke: clinical and research implications. Stroke 2017;48:2621-7. 
25 Broocks G, Rajput F, Hanning U, et al. Highest lesion growth rates in patients with hyperacute stroke. Stroke 2018:STROKEAHA118023457.

26 Boulouis G, Lauer A, Siddiqui AK, et al. Clinical imaging factors associated with infarct progression in patients with ischemic stroke during transfer for mechanical thrombectomy. JAMA Neurol 2017;74:1361-7.
27 Bhatia R, Hill MD, Shobha N, et al. Low rates of acute recanalization with intravenous recombinant tissue plasminogen activator in ischemic stroke: real-world experience and a call for action. Stroke 2010;41:2254-8.

28 Kassem-Moussa H, Graffagnino C. Nonocclusion and spontaneous recanalization rates in acute ischemic stroke: a review of cerebral angiography studies. Arch Neurol 2002;59:1870-3. 